\title{
Processing and properties of high density polyethylene/date palm fiber composites prepared by a laboratory mixing extruder
}

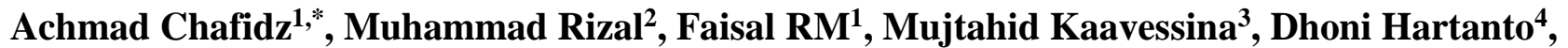 \\ S.M. AIZahrani ${ }^{5}$ \\ ${ }^{1}$ Chemical Engineering Department, Universitas Islam Indonesia, Yogyakarta 55584, Indonesia \\ Phone: +62 274895287 Ext. 119 / Fax: +62 274895007 \\ ${ }^{2}$ PT Petrokimia Gresik, Jalan Jenderal Akhmad Yani, Gresik 61119, INDONESIA \\ ${ }^{3}$ Chemical Engineering Department, Universitas Sebelas Maret, Surakarta 57126, INDONESIA \\ ${ }^{4}$ Chemical Engineering Department, Universitas Negeri Semarang, Semarang 50229, INDONESIA \\ ${ }^{5}$ Chemical Engineering Department, King Saud University, P.O. BOX 800, Riyadh 11421, \\ SAUDI ARABIA \\ *Email: achmad.chafidz@uii.ac.id
}

\begin{abstract}
In this work, "green" composites made from High Density Polyethylene (HDPE) and natural fiber based date palm fiber have been prepared and studied. The effects of different loadings of date palm fibers (DPF) on the morphological, thermal and melt rheological properties of the composites have been investigated. Morphological investigation showed that the fibers were evenly dispersed in HDPE matrix at all DPF loadings. Additionally, the results of differential scanning calorimetry (DSC) analysis revealed that the addition of the DPF in the HDPE matrix has slightly increased the crystallization temperature $\left(\Delta T= \pm 1.33^{\circ} \mathrm{C}\right)$. However, the crystallinity index, $X_{\mathrm{c}}$ of the composites at all DPF loadings were lower than the neat HDPE. The decrease of $X_{\mathrm{c}}$ was approximately $10.5-14 \%$. Differential scanning calorimetry (DSC) analysis results revealed that the addition of the DPF into the HDPE matrix has increased the crystallization temperature. However, the crystallinity index of the composites at all DPF loadings were lower than the neat HDPE. In term of melt rheological analysis, the complex viscosity of the composites were all higher than the HDPE matrix and increased with the increase of DPF loadings, which was due to the restriction of the HDPE chain segment movements as the amount of DPF increased.
\end{abstract}

Keywords: High density polyethylene; date palm fiber; composites; melt blending; thermal properties; rheological properties.

\section{INTRODUCTION}

In the pursuing of more sustainable materials, industries are focusing in the fabrication of materials based on the renewable sources. Ecological and environmental concern, as well as the recyclability have resulted in a great interest in utilization of green materials [1]. Additionally, the needs to look for alternative materials based on renewable/green resources are highly present at the policy-making level. The policy to use many types of natural resources or bio-based materials to make advanced products in 
many applications are highly considered, since it is in accordance with the growing awareness of environmental issues [1-3]. Several visionary concepts, like bio-economy or the green economy have also been introduced and implemented in the strategic planning of industrial companies in Europe countries [4].

One of natural resource or bio-based materials is natural fibers. Natural fibers usually referred to plant fibers. In the history of mankind, these fibers have played important role in daily life with many purposes, such as bamboo and wood, which are particularly used in the construction for long time. There are many types of natural fibers besides bamboo and wood. These fibers can be used to make advanced products like polymer composites. Natural Fiber filled Polymer Composites (NFPCs) can be considered as one of the alternative materials, as mentioned in the previous paragraph. Because, they consist of natural fibers (as filler) added in the polymer matrix, which may also from bio-masses (e.g. PLA) [3]. The Natural Fiber filled Polymer Composites (NFPCs) or simply can be called Natural Fiber Composites (NFCs), recently has highly regarded as valuable materials [5]. Besides polymer composites, natural fibers have also been used in various applications. Fibers from banana, pineapple, sisal, jute, coir, etc. have been used in packaging industries, building, automotive, aerospace, textile, paper, fiber board, etc [6].

Additionally, natural fibers have also been considered as one of important reinforcing materials that could replace synthetic fibers for fabrication of polymer composites. Because they are matching in terms of environmental issues (e.g. biodegradability), recyclability, cost, availability (i.e. renewable materials), and less harmful to health if compared to synthetic fibers, e.g. aramid, glass, carbon fibers [7-11]. In the last few years, the use of natural fibers to substitute the glass fibers as reinforcing materials in composite fabrication has attracted great interest due to the increase of environmental policy and the needs to develop sustainable materials [11]. Furthermore, it can be said that the potentials of natural fibers such as flax, sisal, and hemp are comparable with the synthetic fiber, especially e-glass in terms of their strength (tensile), modulus elasticity, strain at break, availability, and price $[5,12]$.

According to the literature [12], cellulose fibers can be grouped based on their origin. Table 1 shows the classification of cellulose fibers and their samples. The types of fibers that are widely used in the composites fabrication are the bast and leaf parts (the hard fiber). Whereas, fiber plants that are commonly used are coir, ramie, cotton, hemp, jute, sisal, flax, kapok, banana, and henequen [10, 12]. Nowadays, agricultural sources are being considered as valuable source for natural filler materials for the polymer composites production. Research and development of these natural filler might support the progress of socio-economic development for agriculture activities in many of the developing countries [13].

Table 1. Fibers classification based on their origin

\begin{tabular}{ccccccc}
\hline Parts of plant & Leaf, & Seed & Bast & Fruit & Grass & Stalk \\
\hline \multirow{4}{*}{$\begin{array}{c}\text { Examples of } \\
\text { the plants }\end{array}$} & Cantala, & Cotton & Flax, & Coir, & Alfa, & Straw \\
& Henequen, & & Hemp, & Kapok, & Bagasse, & (cereal) \\
& Pineapple, & & Jute, & Oil palm & Bamboo & \\
& Sisal, Banana & & Ramie & & & \\
\end{tabular}

There are numerous research studies on Natural Fiber Polymer Composites (NFPCs), e.g. kenaf fiber composites [7-9, 14, 15], sugar palm fiber [16]; hemp fibers [17]; date palm [18]; flax fibers [11]. Whereas, particular natural fiber that was used in the current study is date palm fiber. The date palm 
tree (Phoenix dactylifera) is normally found in the North Africa, Canary Islands, India, Pakistan, States (i.e. California), and especially Middle East, [19]. Date palm fiber which is abundant in certain areas like the Middle East normally considered as a waste or low value products. Therefore, the use of waste date palm fiber is expected to give advantages in local composite industries by encouraging open new market.

The utilization of date palm fiber as filler has been studied by numerous researchers. Several literatures reported the use of date palm fibers as mortar reinforcement for insulating materials in building [20-23]. Nevertheless, based on our literatures survey, there were few studies about the use of date palm fibers as natural filler in polymeric materials [19, 24-26], and thus make this research field more interesting. Additionally, polymer (in general) can be classified into two types, i.e. thermoplastics and thermosets. The thermoplastic is the most widely used polymer matrix to fabricate polymer composites. The commonly used thermoplastic for polymer composites is polyethylene (PE), polypropylene (PP), polystyrene (PS), polyvinyl chloride (PVC), etc [1, 10]. Therefore, a High Density Polyethylene (HDPE) was used in the present work.

A laboratory mixing extruder (LME) is a small-scale mixing extruder. The use of this kind of mixing extruder instead of high-scale mixing extruder to make polymer composites, especially during the formulation of right composition (polymer matrix: filler) or simply as a preliminary experimental design can be considered as a good idea. Because this LME only requires small amount of materials, thus it can reduce the cost of composites preparation for the purposes mentioned above. Our literatures survey revealed that the studies of polypropylene/date palm fiber composites using a lab scale mixing extruder to prepare polymer composites is very limited compared to the use of large-scale mixing extruder, and thus it is an interesting topic of research. The aims of this work are to fabricate high density polyethylene (HDPE)/date palm fiber (DPF) composites through melt blending method using a Laboratory Mixing Extruder (LME) and to investigate the morphology, thermal, and melt rheological properties of the prepared composites.

\section{EXPERIMENTAL}

\section{Materials}

Date palm fibers used in this work were obtained by local farm. The date palm fiber (DPF) used in this study was extracted from the stem. The DPFs were cleaned, sorted, and separated from impurities (e.g. sand, dirt, etc.), then cut using high-speed chopper (Backerson BS009002, Mexico) and screened through mesh sieve to obtain fractions $2.34 \mathrm{~mm}$ size. The fiber length took in this study was approximately $2 \mathrm{~mm}$ length as it fulfilled the standard Tyler screen mesh that available in the laboratory. Furthermore, the fibers dried at $70^{\circ} \mathrm{C}$ for 24 hours to reduce the moisture content. Observation of representative fibers via SEM micrographs revealed that the aspect ratio (L/D) of the DPF was approximately 4.16. An injection molding grade High Density Polyethylene (HDPE) (product name: M-300054) used as a polymer matrix was obtained from SABIC plastic industry in Saudi Arabia. The HDPE has lower melting point among plastics which make them processable at temperature below the degradation temperature of natural fibers. The melt flow index (MFI) of the HDPE is $30 \mathrm{~g} / 10 \mathrm{~min}$, which is low melt viscosity, thus makes it easy to be mixed with the fiber. The photographs of the HDPE pellets and the data palm fiber are shown in Figure 1. 


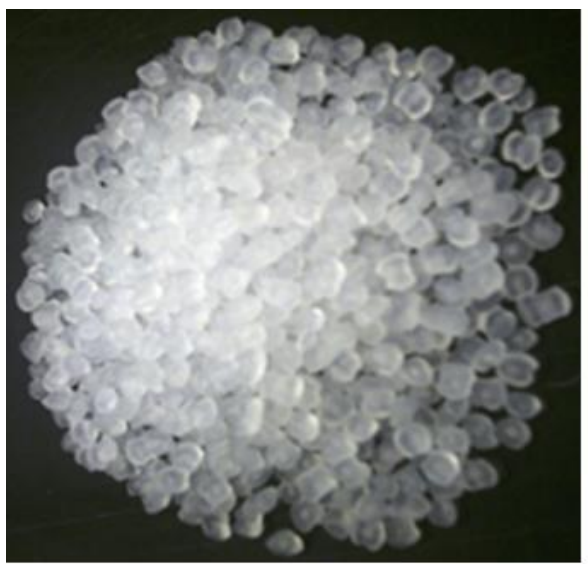

(a)

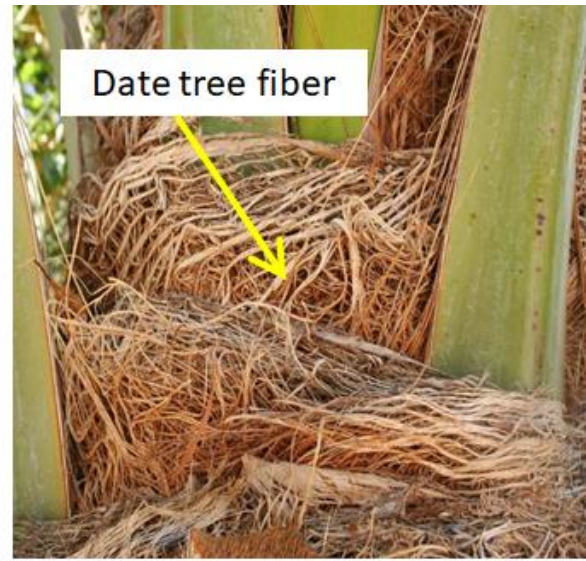

(b)

Figure 1. The photographs of materials used in the preparation of HDPE/DPF composites: a) HDPE pellets; and b) Date palm fiber.

\section{Experimental Procedure}

To prepare the composites, the HDPE and DPFs were melt compounded and pelletized with different loadings of date palm fibers ranging from 5\% to 30\% using a Laboratory Mixing Extruder (LME), Dynisco-USA. Figure 2 shows the photograph of the LME complete with the pelletizer and ribbon maker. The LME is a laboratory scale mixing extruder with diameter of the rotor and barrel, approximately $18.8 \mathrm{~mm}$ and $19 \mathrm{~mm}$, respectively. Inside the barrel is a smooth rotating shaft (see Figure 2) [27].

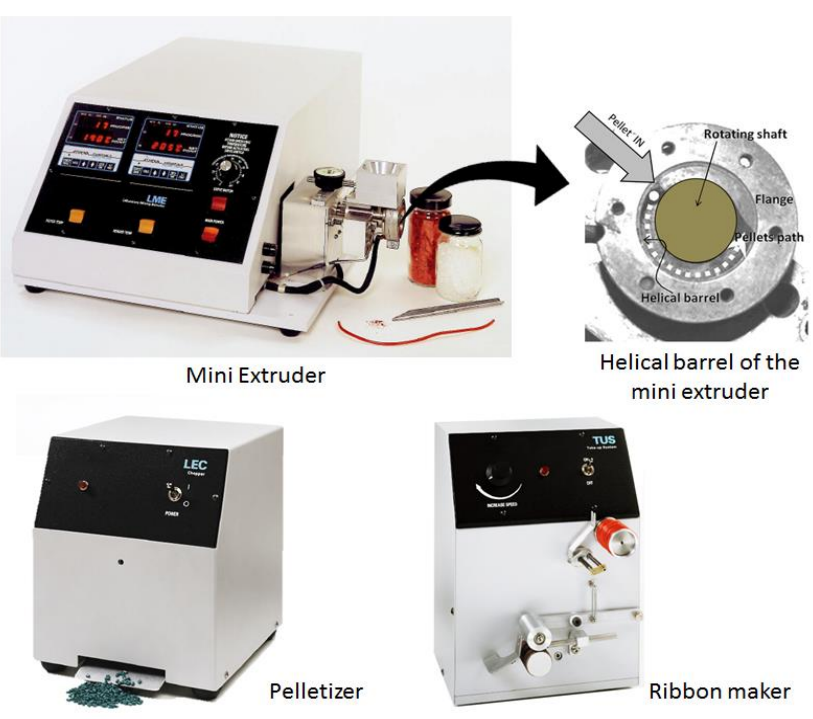

Figure 2. Laboratory Mixing Extruder complete with its pelletizer and ribbon maker.

The preparation of the composites was done in two steps. The first step, the HDPE pellets and the prepared short DPF fiber were manually mixed, and then they were melt blended with the LME to make HDPE/DPF composites. The processing conditions of the LME were as follow: the barrel temperature $=260^{\circ} \mathrm{C}$, die temperature $=265^{\circ} \mathrm{C}$, and rotor speed $=250 \mathrm{rpm}$. The extruded composites 
from the LME was subsequently cooled in the water bath and then pelletized by using the pelletizer. Afterward, the wet pellets were dried in the laboratory oven at $70^{\circ} \mathrm{C}$ for about 24 hours to remove the water/moisture. The second step, the dried composites pellets were secondly melt blended again in the LME with the same processing conditions. The extruded composites were subsequently fed into the ribbon maker to make ribbon form of the composites. The schematic diagram of the preparation of the composites is exhibited in Figure 3. Whereas, the list of the composites samples fabricated in this study is shown in Table 2.

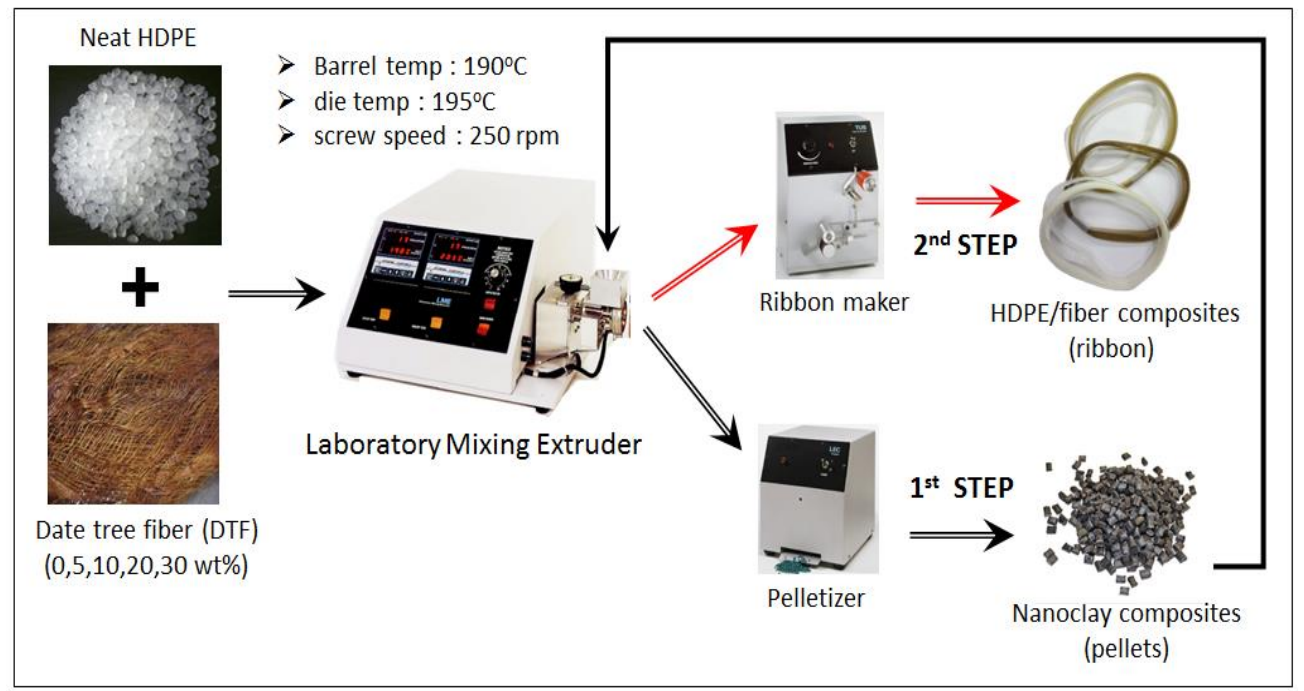

Figure 3. The schematic diagram of the preparation of the HDPE/DPF composites.

Table 2. Nomenclature of HDPE/DPF composites samples.

\begin{tabular}{ccc}
\hline Sample Name & HDPE (wt \%) & DPF (wt \%) \\
\hline DC-0-LME & 100 & 0 \\
DC-5-LME & 95 & 5 \\
DC-10-LME & 90 & 10 \\
DC-20-LME & 80 & 20 \\
DC-30-LME & 70 & 30 \\
\hline
\end{tabular}

\section{Characterization of composites}

The surface morphology of the composites samples was investigated by using a scanning electron microscope (SEM), JSM-6360A, JEOL, Japan. The samples used were the ribbon samples prepared by the LME and its ribbon maker (see Figure 3). Prior to the SEM analysis, the composites samples were finely coated by using a sputtering process with a thin layer of highly conductive metal (i.e. gold) to minimize electronic charging and overheating of the composites samples [28]. Figure 4a shows the photograph of the SEM. 


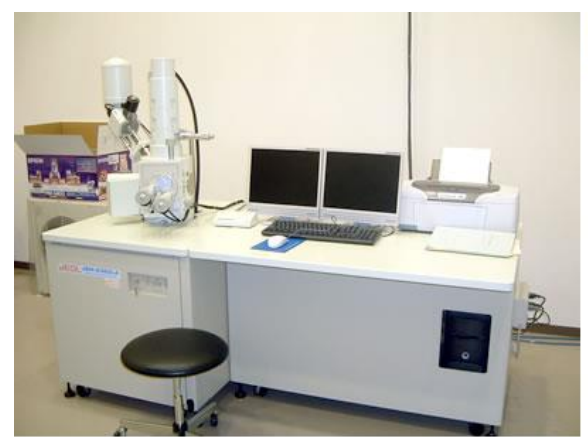

(a)

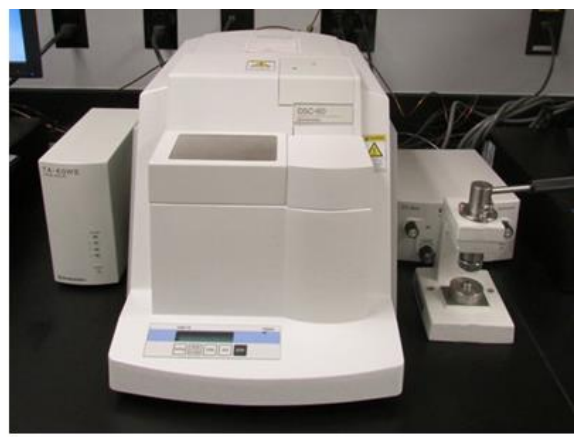

(b)

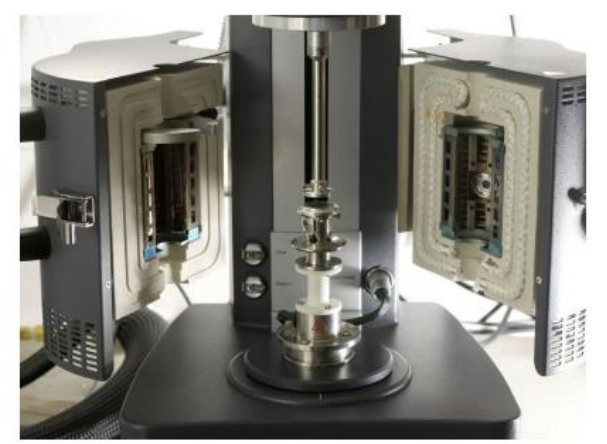

(c)

Figure 4. Photographs of apparatus used to characterize the prepared HDPE/DPF composites: a) Scanning Electron Microscopy (SEM); b) Differential Scanning Calorimetry (DSC); and c) Oscillatory Rheometer

To investigate the thermal characteristics of the composites samples, a differential scanning calorimeter (DSC) model DSC-60, Shimadzu, Japan was used (see Figure 4b). In the DSC, the composite pellets (approximately $4-7 \mathrm{mg}$ ) were weighted and put in a typical aluminum pan, and then sealed. As a reference, an empty sealed aluminum pan was analyzed together with sample in the DSC. All the DSC analyses were carried out non-isothermally in air environment. The procedure of the DSC analysis is explained as follows [27, 28]: The composites pellets were heated at constant heating rate of $10^{\circ} \mathrm{C} / \mathrm{min}$, from $30^{\circ} \mathrm{C}$ to $200^{\circ} \mathrm{C}$ and held for $5 \mathrm{~min}$ in order to remove their thermal history and to ensure the crystals are completely melted. This step is called $1^{\text {st }}$ heating scan. Afterward, the composites samples were cooled down to $30^{\circ} \mathrm{C}$ with the same rate (i.e. $-10^{\circ} \mathrm{C} / \mathrm{min}$ ) to investigate the crystallization behavior of the composites. This step is called cooling scan. Finally, they were heated for the second time $\left(2^{\text {nd }}\right.$ heating scan $)$ at the same rate of $10^{\circ} \mathrm{C} / \mathrm{min}$ up to $300^{\circ} \mathrm{C}$ to find the melting temperature of each composites. Figures $5 \mathrm{a}$ and $5 \mathrm{~b}$ show the typical DSC thermograms of the HDPE/DPF composites with the time and temperature dependence, respectively. The DSC thermograms were used to investigate the thermal properties are as follow: The $2^{\text {nd }}$ heating scan for studying the melting temperature and crystallinity index; and the cooling scan for studying the crystallization temperature of the HDPE/DPF composites (see Figure 5b).

The melt rheological behavior of the HDPE/DPF composites were analyzed using an oscillatory rheometer instrument, ARG2 from TA Instruments, USA. The melt rheological analysis was conducted under torsion mode using a parallel plate configuration (i.e. diameter $=25 \mathrm{~mm}$ and constant gap $=2$ $\mathrm{mm}$ ). The composites pellets from the pelletizer (after dried in the oven) were used for this test. The 
experiments were carried out under frequency sweep mode at fixed temperature of $150^{\circ} \mathrm{C}$ and oscillation stress of $1 \mathrm{~Pa}$, with angular frequency in the range of $1-628.3 \mathrm{rad} / \mathrm{s}$. Prior to the test, the excess of composites melts in the edge of parallel plates were cleaned to validate the analysis [29]. The photograph of the oscillatory rheometer is shown in Figure 4c. The result of the rheological test was a plot of dynamic complex viscosity $\left|\eta^{*}\right|$ vs angular frequency $(\omega)$.
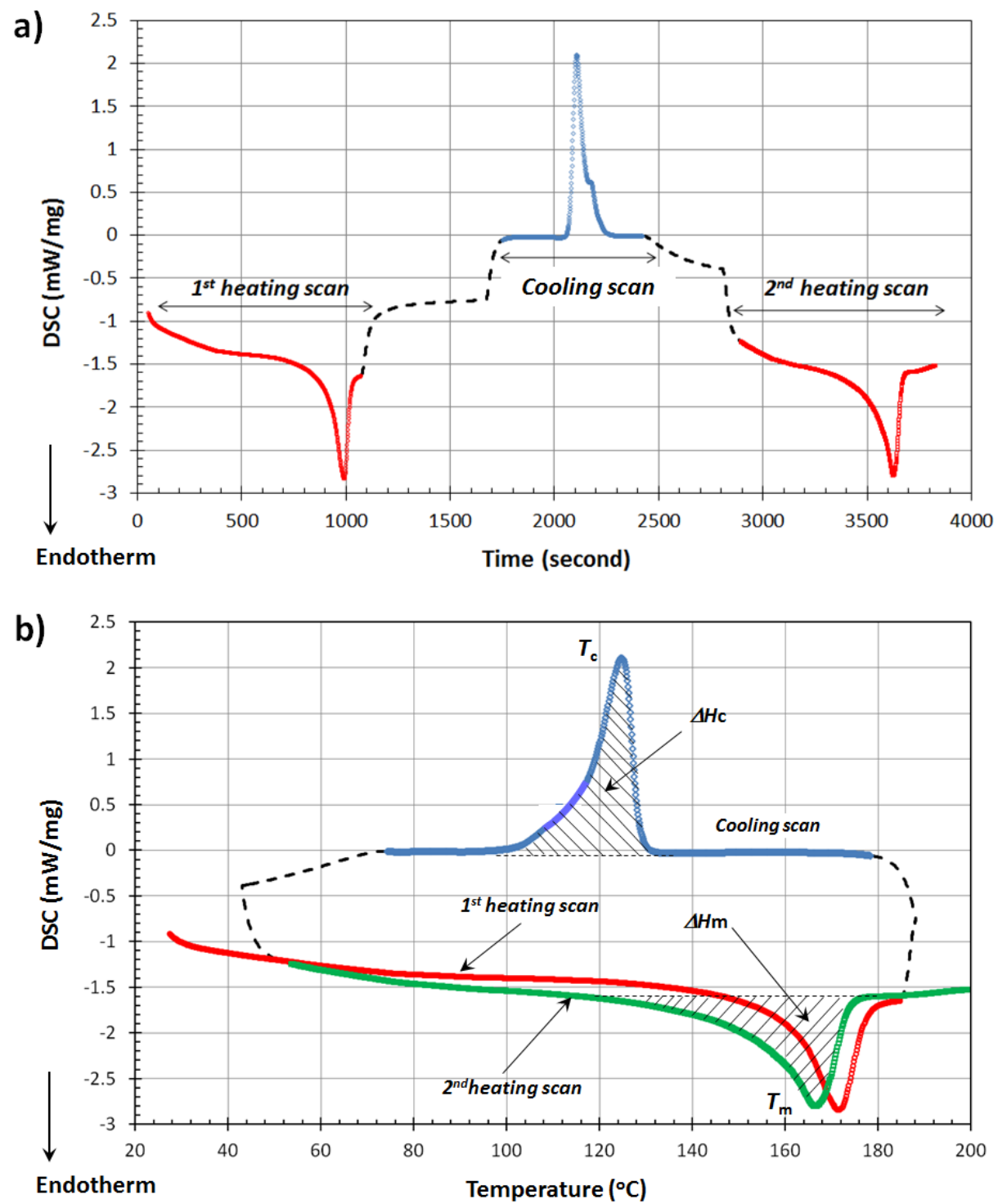

Figure 5. Typical DSC thermograms of the HDPE/DPF composites: a) Time dependence; and b) Temperature dependence. 


\section{RESULTS AND DISCUSSION}

The scanning electron microscopy (SEM) micrograph of the date palm fiber (DPF) is shown in Figure 6a. As seen in the figure, the length of the fiber used in this work was approximately $2 \mathrm{~mm}$, as it followed the standard Tyler screen that available in the laboratory. Whereas, the SEM micrographs of the HDPE/DPF composites samples (ribbon) are shown in Figures 6b-6d. As seen in Figure 6b, there were some of fibers embedded in the composites ribbon sample (i.e. DC-5 LME). There were also some of small pores on the surface of the ribbon. The pores were likely due to some of air trapped during the melt mixing of the composites. As seen in the figure, the fiber was well distributed in the surface of the ribbon. Additionally, some of fibers were also observable in Figure 6c. If compared to other literature [25], the dispersion of DPF particles in this was work not good enough, which was likely due to the high loading percentage of the fibers and the limitation of the Laboratory Mixing Extruder (LME).
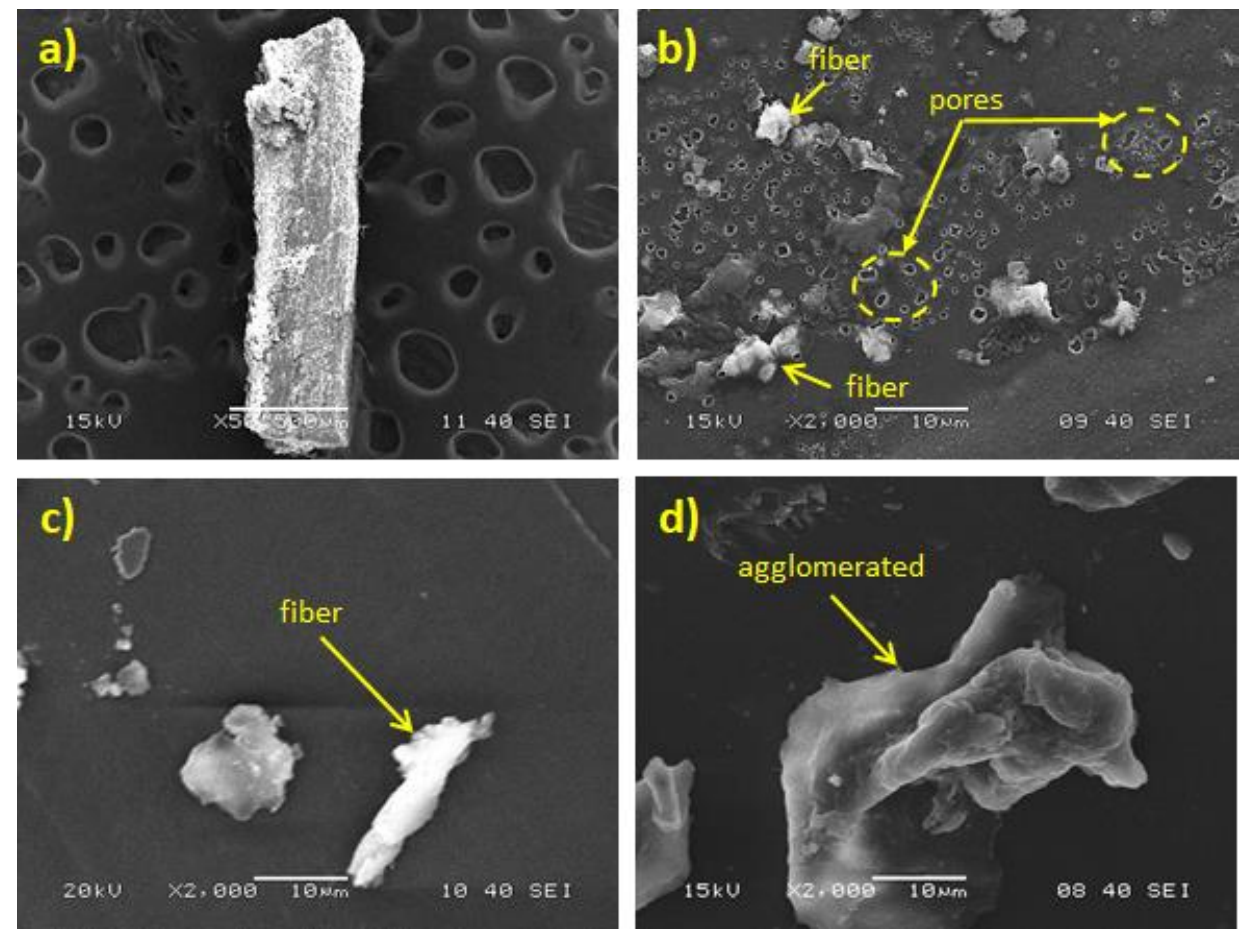

Figure 6. SEM micrographs (magnification 2000X) of the HPDE/DPF composites ribbon surfaces at different loadings: a) DC-5 LME; b) DC-10 LME; and c) DC-30 LME.

In general, the fibers in the composites at all DPF loading levels were evenly distributed. Nevertheless, all the fibers in the HDPE matrix (as seen in Figures $6 b, 6 c$, and $6 \mathrm{~d}$ ), some of their parts were embedded in the composites ribbon, and the remaining parts were appeared on the surface of the composites ribbon. This was likely due to the size of the ribbon made by the LME and its ribbon maker, in which the ribbon was relatively thin. In our point of view, this was indeed the limitation of the use of a Laboratory Mixing Extruder (LME). Nevertheless, using the LME to prepare date fiber based composites was a good research experience. This study can be used as a preliminary step for the 
next work with the higher scale of extruder for preparing the composites. Whereas, the adhesion or interaction between the DPF and the HDPE matrix could not be observed in the SEM micrographs. Enhancing the adhesion or compatibility level between the fiber and the matrix usually can be done by chemical treatment of the fiber surface as well as the matrix, which was not done in the present work. This will be a good recommendation for the future work.

\section{Thermal Properties}

The differential scanning calorimetry (DSC) apparatus was utilized to investigate the melting and crystallization behavior of the HDPE/DPF composites. As explained in the experimental section, the DSC thermograms used for the melting analysis was the $2^{\text {nd }}$ heating scan, whereas for the crystallization analysis was the cooling scan. Figure 7 shows the DSC thermograms of the $2^{\text {nd }}$ heating scan procedure (i.e. melting analysis). Some parameters that could be derived from these DSC thermograms are crystallization temperature $\left(T_{\mathrm{c}}\right)$, melting temperature $\left(T_{\mathrm{m}}\right)$, heat of fusion $(\Delta H)$, and crystallinity index $\left(X_{\mathrm{c}}\right)$ and are listed in Table 3. The melting temperature, $T_{\mathrm{m}}$ was taken as the highest/peak temperature of the DSC melting endotherm [30]. The crystallinity index of the composites can be determined from the heat of fusion $\left(\Delta H_{\mathrm{m}}\right)$ of DSC analysis and value of $100 \%$ of crystalline polymer material taken from the literature $\left(\Delta H_{\mathrm{m}}{ }^{\circ}\right)$ [31]. In which, the $\Delta H_{\mathrm{m}}{ }^{\circ}$ of the HDPE is approximately $292.6 \mathrm{~J} / \mathrm{g}$. Eq. (1) below was used to calculate the crystallinity index $\left(X_{\mathrm{c}}\right)$ of the HDPE/DPF composites [32-34]:

$$
X_{C}=\frac{\Delta H_{m}}{\Delta H_{m}^{o}} \cdot 100[\%]
$$

Figure 7 and Table 3 show that the melting temperature, $T_{\mathrm{m}}$ of the composites are similar to that of the $T_{\mathrm{m}}$ of the neat HDPE (i.e. $130^{\circ} \mathrm{C}$ ). It can be implied that the $T_{\mathrm{m}}$ of the composites were not considerably affected by the addition of date palm fiber. The crystallinity index of the HDPE/DPF composites is listed in Table 3. The crystallinity index of the composites at all DPF loadings was lower than the neat PP. The decrease of $X_{\mathrm{c}}$ was approximately $10.5-14 \%$. This result is also similar to other literature, in which there was also a decrease in $X_{\mathrm{c}}$ [35]. The decreasing crystallinity index by the addition of DPF was likely due to the restricted movement of the HDPE molecular chains by the presence of the, which also reduced the spaces/free volume available to be occupied by the molecular chains. Thus inhibiting the crystal growth and alignment of the crystal lattice, which consequently reduced the crystallinity index $[31,33]$. 


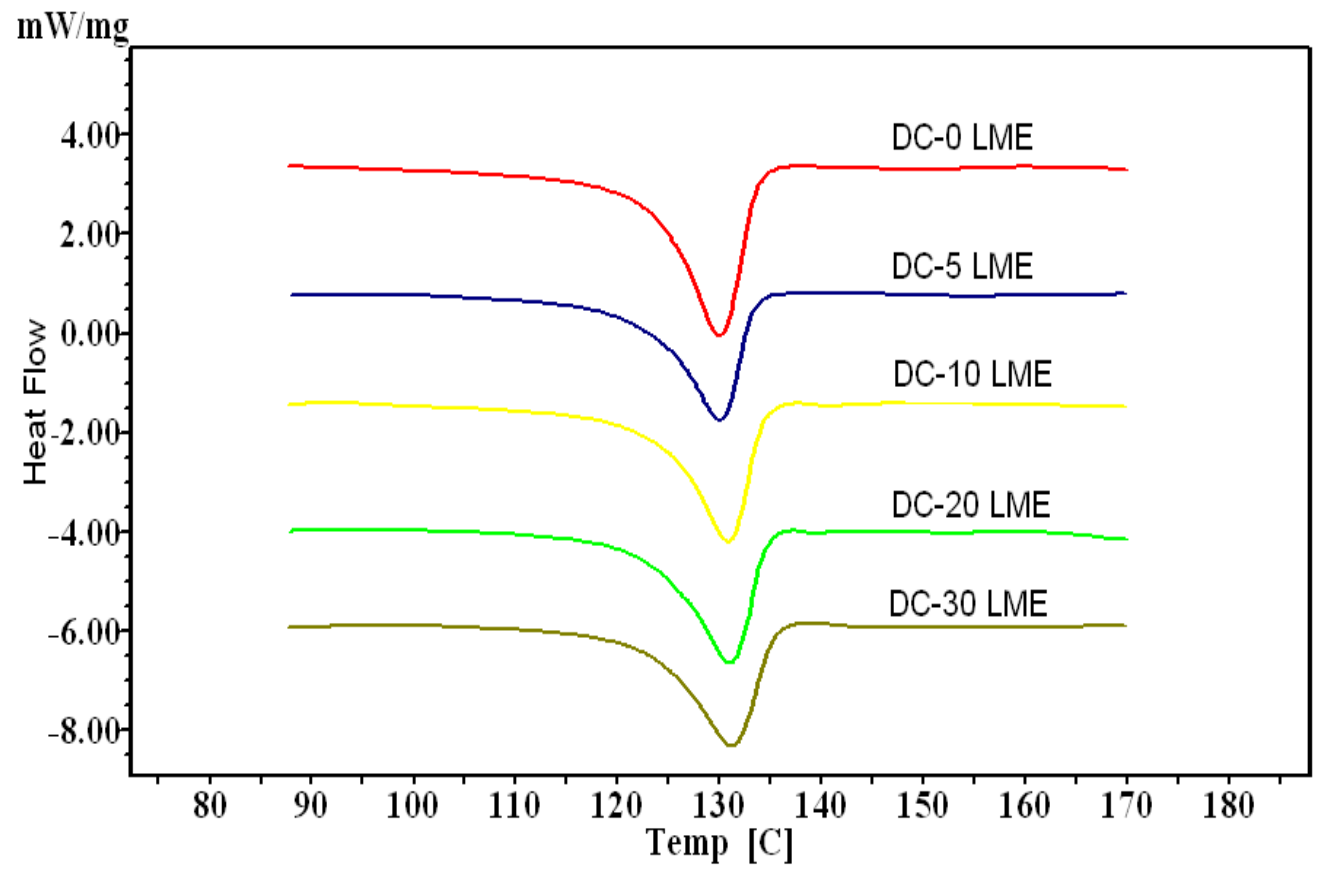

Figure 7. DSC melting thermograms of HDPE/DPF composites. (Note: adjustment on the ydirection has been applied for convenience).

Table 3. $T_{\mathrm{m}}, T_{\mathrm{c}}$, and $X_{\mathrm{c}}$ of HDPE/DPF composites prepared by LME

\begin{tabular}{ccccc}
\hline $\begin{array}{c}\text { Sample } \\
\text { Name }\end{array}$ & $\begin{array}{c}T_{\mathrm{m}}\left({ }^{\circ} \mathrm{C}\right) \\
\pm 0.2 \%\end{array}$ & $\begin{array}{c}T_{\mathrm{c}}\left({ }^{\circ} \mathrm{C}\right) \\
\pm 0.2 \%\end{array}$ & $\begin{array}{c}\Delta H(\mathrm{~J} / \mathrm{g}) \\
\pm 2 \%\end{array}$ & $\begin{array}{c}X_{\mathrm{c}}(\%) \\
\pm 2 \%\end{array}$ \\
\hline DC-0 LME & 130.0 & 115.4 & 166.1 & 56.77 \\
DC-5 LME & 130.0 & 115.9 & 130.1 & 44.46 \\
DC-10 LME & 130.8 & 116.0 & 135.7 & 46.38 \\
DC-20 LME & 130.9 & 116.7 & 135.5 & 46.31 \\
DC-30 LME & 131.2 & 116.3 & 125.9 & 43.03 \\
\hline
\end{tabular}

Additionally, Figure 8 shows the DSC crystallization thermograms of HDPE/DPF composites taken from the DSC cooling scan step (see Figure 5b). Whereas, the value of crystallization temperature, $T_{\mathrm{c}}$ of the composites are listed in Table 3. Generally, as seen in the table, the addition of fibers leads to the slight increase of crystallization temperature $\left(\Delta T= \pm 1.33{ }^{\circ} \mathrm{C}\right)$. This result is similar to other literature [35], in which there was also an increase in Tc, approximately $1-3{ }^{\circ} \mathrm{C}$ ). As seen in Table 3, the highest increase of $T_{\mathrm{c}}$ was occurred at DPF loading of $20 \mathrm{wt} \%$ (i.e. DC-20-LME). The $T_{\mathrm{c}}$ of the composites sample, DC-20-LME increased from 115.4 to $116.7^{\circ} \mathrm{C}$. At higher content, the trend of $T_{\mathrm{c}}$ decreased but still higher than the neat HDPE. The increase of $T_{\mathrm{c}}$ could be possibly due to the presence of natural DPF fibers which acted as nucleating agent for the crystallization process of the HDPE matrix $[32,35]$. The higher the $T_{\mathrm{c}}$ value indicating the higher crystallization rate. Because, the 
crystallization or solidification process of the composites melt could start earlier. This fact can be considered as an economic advantage. Because, during the injection molding, the molded parts of the composites would start to solidify earlier, which shorten the cycling time of the injection molding process, meaning time-saving (i.e. economic advantage) [36].

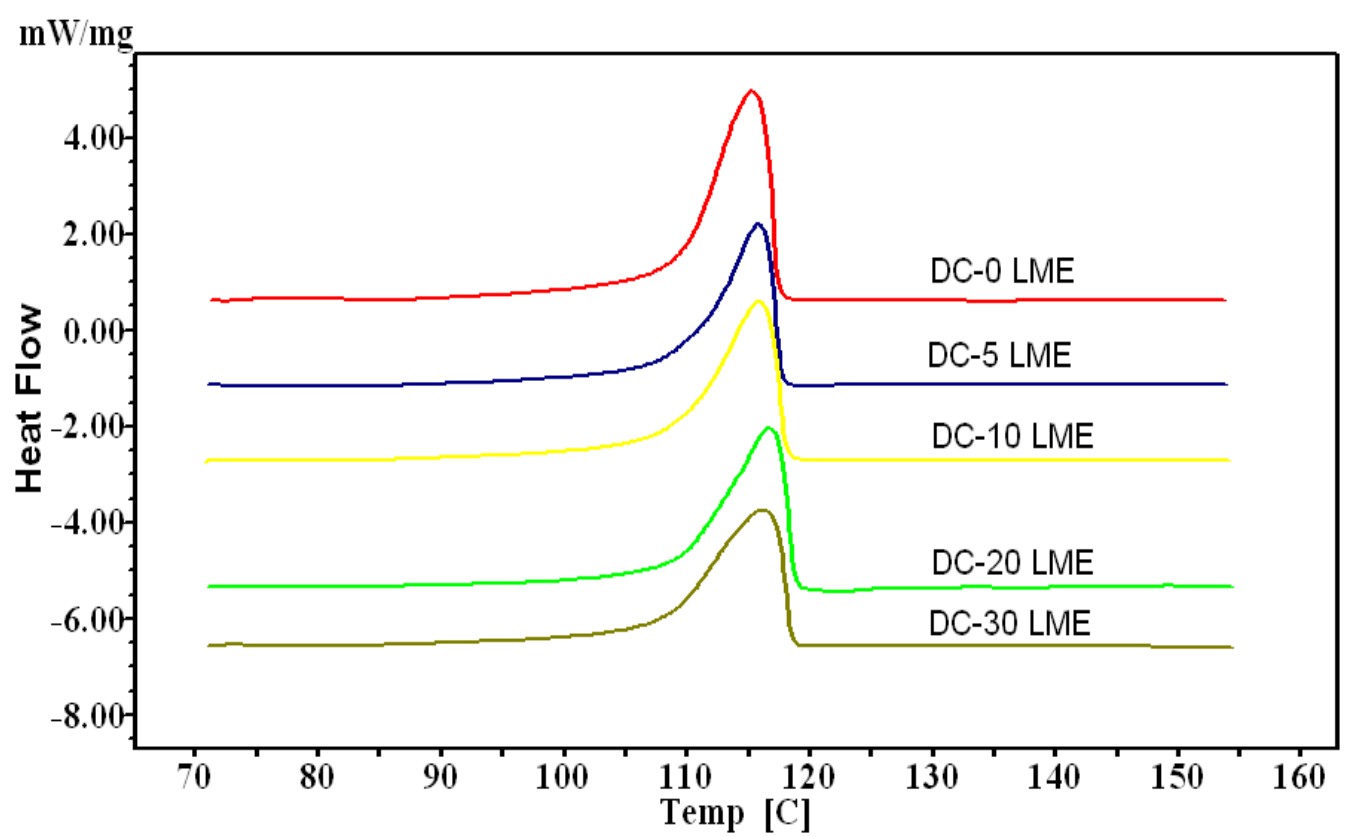

Figure 8. DSC crystallization thermograms of HDPE/DPF composites. (Note: adjustment on the $\mathrm{y}$-direction has been applied for convenience).

\section{Melt rheological properties}

The melt rheological analysis was carried out to investigate the effect of DPF loadings in the flow behavior or deformation behavior of the HDPE/DPF composites when it is being processed [37]. The melt rheological properties were characterized by using a dynamic frequency sweep test with parallel plate geometry. The test was performed at constant temperature (i.e. $150{ }^{\circ} \mathrm{C}$ ), oscillation stress (i.e. 1 $\mathrm{Pa}$ ), and variable angular frequencies (i.e. $1-628.3 \mathrm{rad} / \mathrm{s}$ ). Figure 9 exhibits the complex viscosity, $\left|\eta^{*}\right|$ versus angular frequency of the composites at different DPF loadings. As seen in the figure, all the samples show the same trend, where the complex viscosity decreased with the increase of angular frequency. Additionally, the complex viscosity of the HDPE/DPF composites was much higher than the neat HDPE and increased with the increase of DPF loadings at all frequency region. This phenomenon was likely due to the restriction of the HDPE chain segment movements as the amount of DPF increased. The similar finding was also mentioned in other literature [37]. Furthermore, as seen in Figure 9, the increase of complex viscosity was more pre-dominant at lower angular frequencies than at higher angular frequencies region. It is because at higher angular frequencies, the fibers will align strongly along with the matrix. Therefore, the probability of fiber-fiber collision was much lower, and thus the increase in the complex viscosity with the DPF loadings was less at higher angular 
frequencies. Additionally, at low frequency region, the decrease (i.e. the slope) of complex viscosity of the composites at higher DPF loadings (i.e. 20 and $30 \mathrm{wt} \%$ ) was very steep if compared to the low DPF loadings. This indicates a shear-thinning behavior of the composites, which also represent the changed behavior from liquid-like to pseudo-like behavior [37].

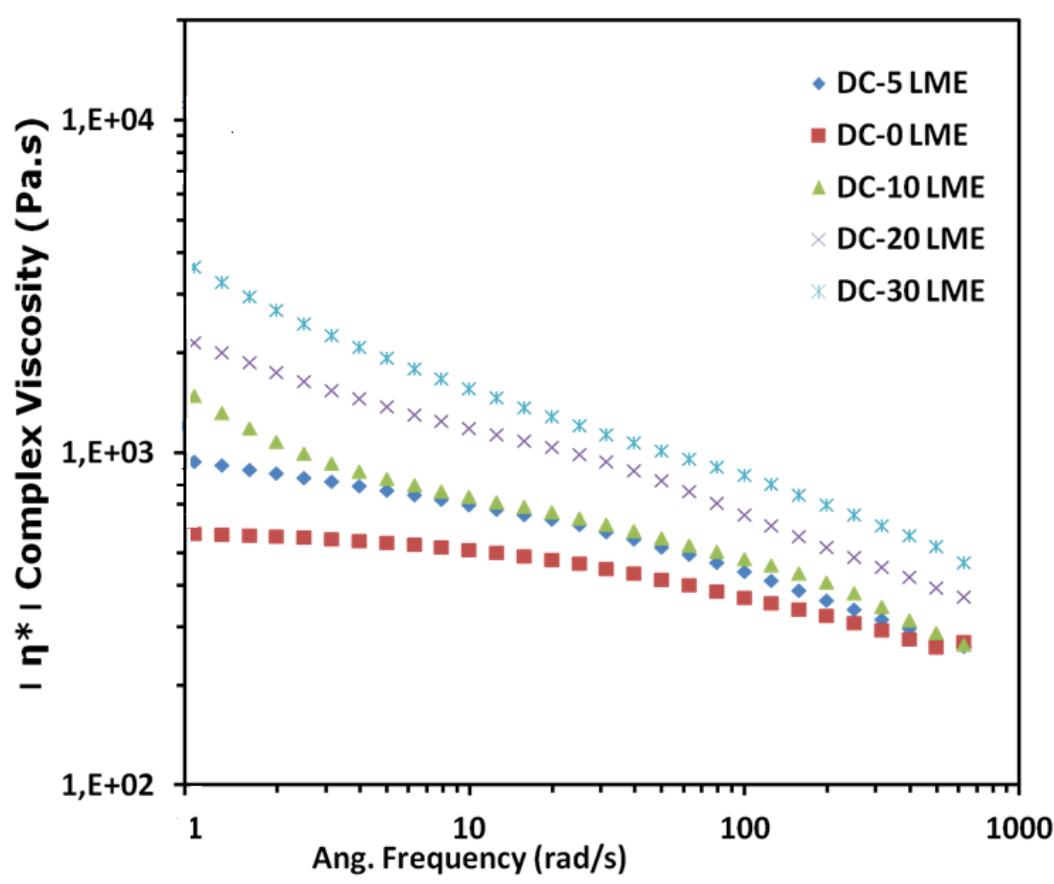

Figure 9. Complex Viscosity of Date palm fiber Composites prepared by LME

\section{CONCLUSIONS}

The morphological, thermal, and melt rheological properties of the HDPE/date palm fiber (DPF) composites have been investigated. The composites were prepared at different DPF loadings (i.e. 5, 10, 20, and $30 \mathrm{wt} \%$ ) via melt blending technique using a Laboratory Mixing Extruder (LME) instrument. Morphological investigation of the composites showed that the fibers in the composites at all DPF loading levels were evenly distributed. Nevertheless, some parts of the fibers were embedded in the composites ribbon, and the remaining parts were appeared on the surface of the composites ribbon. This was likely due to the size of the ribbon made by the LME and its ribbon maker, in which the ribbon was relatively thin. Additionally, the differential scanning calorimetry (DSC) analysis results revealed that the addition of the DPF into the HDPE matrix has increased the crystallization temperature. However, the crystallinity index of the composites at all DPF loadings was lower than the neat HDPE, which could be related to the hindered movement of the HDPE molecular chains by the presence of the fibers. Whereas, the melting temperature was not considerably affected by the presence of the DPF. In the melt rheological study, the HDPE/DPF composites showed higher complex 
viscosity, $\left|\eta^{*}\right|$ than the neat HDPE, and increased with the increasing DPF loadings, which was due to the restriction of the HDPE chain segment movements as the amount of DPF increased.

\section{ACKNOWLEDGEMENTS}

The authors would like to thank Center of Excellence for Research in Engineering Materials (CEREM), College of Engineering, King Saud University for their financial supports.

\section{REFERENCES}

[1] Väisänen T, Das O, Tomppo L. A review on new bio-based constituents for natural fiber-polymer composites. Journal of Cleaner Production. 2017;149: 82-96.

[2] Winandy JE, Williams RS, Rudie AW, Ross RJ. Opportunities for using wood and biofibers for energy, chemical feedstocks, and structural applications, A2 - Pickering, Kim L. Properties and Performance of Natural-Fibre Composites. Woodhead Publishing. 2008;p. 330-55.

[3] Väisänen T, Haapala A, Lappalainen R, Tomppo L. Utilization of agricultural and forest industry waste and residues in natural fiber-polymer composites: A review. Waste Management. 2016;54:62-73.

[4] McCormick K, Kautto N. The bioeconomy in Europe: An overview. Sustainability. 2013; 5(6):2589-608.

[5] Al-Oqla FM, Sapuan SM. Natural fiber reinforced polymer composites in industrial applications: feasibility of date palm fibers for sustainable automotive industry. Journal of Cleaner Production. 2014;66:347-54.

[6] Kiruthika AV. A review on physico-mechanical properties of bast fibre reinforced polymer composites. Journal of Building Engineering. 2017;9:91-99.

[7] Fairuz A, Sapuan SM, Zainudin E, Jaafar C. Effect of filler loading on mechanical properties of pultruded kenaf fibre reinforced vinyl ester composites. Journal of Mechanical Engineering and Sciences. 2016;10:1931-42.

[8] Ismail A.E, Aziz CA, Aswadi M. Tensile strength of woven yarn kenaf fiber reinforced polyester composites. Journal of Mechanical Engineering and Sciences. 2015;9:1695-704.

[9] Loh X, Daud MM, Selamat M. Mechanical properties of kenaf/polypropylene composite: An investigation. Journal of Mechanical Engineering and Sciences. 2016;10(2):2098-2110

[10] $\mathrm{Ku} \mathrm{H,Wang} \mathrm{H,} \mathrm{Pattarachaiyakoop} \mathrm{N,} \mathrm{Trada} \mathrm{M.} \mathrm{A} \mathrm{review} \mathrm{on} \mathrm{the} \mathrm{tensile} \mathrm{properties} \mathrm{of} \mathrm{natural} \mathrm{fiber}$ reinforced polymer composites. Composites Part B - Engineering. 2011;42(4):856-73.

[11] Yan L, Chouw N, Jayaraman K. Flax fibre and its composites - A review. Composites Part B Engineering. 2014;56:296-317.

[12] Pilla S. Handbook of bioplastics and biocomposites engineering applications. John Wiley \& Sons. 2011.

[13] Saba N, Tahir PM, Jawaid M. A review on potentiality of nano filler/natural fiber filled polymer hybrid composites. Polymers. 2014;6(8):2247-73. 
[14] Fauzi F, Ghazalli Z, Siregar J. Effect of various kenaf fiber content on the mechanical properties of composites. Journal of Mechanical Engineering and Sciences. 2016;10:2226-33.

[15] Lee C, Sapuan SM, Lee J, Hassan M. Mechanical properties of kenaf fibre reinforced floreon biocomposites with magnesium hydroxide filler. Journal of Mechanical Engineering and Sciences. 2016;10(3):2234-48

[16] Mohammed A, Bachtiar D, Siregar J, Rejab M. Effect of sodium hydroxide on the tensile properties of sugar palm fibre reinforced thermoplastic polyurethane composites. Journal of Mechanical Engineering and Sciences. 2016;10(1):1765-77.

[17] Pickering KL, Beckermann GW, Alam SN. Foreman N.J. Optimising industrial hemp fibre for composites. Composites Part A - Applied Science. 2007;38(2):461-68.

[18] Ibrahim H, Farag M, Megahed H, Mehanny S. Characteristics of starch-based biodegradable composites reinforced with date palm and flax fibers. Carbohydrate Polymers. 2014;101:11-19.

[19] Alawar A, Hamed AM, Al-Kaabi K. Characterization of treated date palm tree fiber as composite reinforcement. Composites Part B - Engineering. 2009;40(7):601-06.

[20] Benmansour N, Agoudjil B, Gherabli A, Kareche A, Boudenne A. Thermal and mechanical performance of natural mortar reinforced with date palm fibers for use as insulating materials in building. Energy and Buildings. 2014;81:98-104.

[21] Chikhi M, Agoudjil B, Boudenne A, Gherabli A. Experimental investigation of new biocomposite with low cost for thermal insulation. Energy and Buildings. 2013;6:267-73.

[22] Boumhaout M, Boukhattem L, Hamdi H, Benhamou B, Ait Nouh F. Thermomechanical characterization of a bio-composite building material: Mortar reinforced with date palm fibers mesh. Construction and Building Materials. 2017;135:241-50.

[23] Boukhattem L, Boumhaout M, Hamdi H, Benhamou B, Ait Nouh F. Moisture content influence on the thermal conductivity of insulating building materials made from date palm fibers mesh. Construction and Building Materials. 2017;148:811-23.

[24] Al-Khanbashi A, Al-Kaabi K, Hammami A. Date palm fibers as polymeric matrix reinforcement: Fiber characterization. Polymer Composites. 2005;26(4):486-97.

[25] Al-Kaabi K, Al-Khanbashi A, Hammami A. Date palm fibers as polymeric matrix reinforcement: DPF/polyester composite properties. Polymer Composites. 2005;26(5):604-13.

[26] Oushabi A, Sair S, Abboud Y, Tanane O, Bouari AE. An experimental investigation on morphological, mechanical and thermal properties of date palm particles reinforced polyurethane composites as new ecological insulating materials in building. Case Studies in Construction Materials. 2017; 7:128-37.

[27] Chafidz A, Kaavessina M, Al-Zahrani S, Al-Otaibi M. Polypropylene/organoclay nanocomposites prepared using a Laboratory Mixing Extruder (LME): crystallization, thermal stability and dynamic mechanical properties. Journal of Polymer Research. 2014;21(6):1-18.

[28] Chafidz A, Latief FH, Samad UA, Ajbar A, Al-Masry W. Nanoindentation creep, nano-impact, and thermal properties of multiwall carbon nanotubes-polypropylene nanocomposites prepared via melt blending. Polymer-Plastics Technology and Engineering. 2016;55(13):1373-85.

[29] Chafidz A, Rengga WDP, Khan R, Kaavessina M, Almutlaq AM, Almasry WA, Ajbar A, Polypropylene/multiwall carbon nanotubes nanocomposites: Nanoindentation, dynamic mechanical, and electrical properties. Journal of Applied Polymer Science. 2017;134:45293. 
[30] Ibrahim MM, Dufresne A, El-Zawawy WK, Agblevor FA. Banana fibers and microfibrils as lignocellulosic reinforcements in polymer composites. Carbohydrate Polymers. 2010;81(4):81119.

[31] Chafidz A, Kaavessina M, Al-Zahrani S, Al-Otaibi MN. Rheological and mechanical properties of polypropylene/calcium carbonate nanocomposites prepared from masterbatch. Journal of Thermoplastic Composite Materials. 2014;29:593-622.

[32] López-Manchado MA, Arroyo M. Thermal and dynamic mechanical properties of polypropylene and short organic fiber composites. Polymer. 2000;41(21):7761-67.

[33] Sui G, Fuqua MA, Ulven CA, Zhong WH. A plant fiber reinforced polymer composite prepared by a twin-screw extruder. Bioresource Technology. 2009;100(3):1246-51.

[34] Amash A, Zugenmaier P. Thermal and dynamic mechanical investigations on fiber-reinforced polypropylene composites. Journal of Applied Polymer Science. 1997;63(9):1143-54.

[35] Mi Y, Chen X, Guo Q. Bamboo fiber-reinforced polypropylene composites: Crystallization and interfacial morphology. Journal of Applied Polymer Science. 1997;64(7):1267-73.

[36] Chafidz A, Hamdan Latief F, Al-Fatesh AS, Kaavessina M. Crystallization and thermal stability of polypropylene/multi-wall carbon nanotube nanocomposites. Philosophical Magazine Letters. 2016;96(10):367-74.

[37] Salleh FM, Hassan A, Yahya R, Azzahari AD. Effects of extrusion temperature on the rheological, dynamic mechanical and tensile properties of kenaf fiber/HDPE composites. Composites Part B - Engineering. 2014;58:259-66. 